Rapid Reviews COVID-19

\title{
Review 2: "Assessment of Airborne Disease Transmission Risk and Energy Impact of HVAC Mitigation Strategies"
}

Marwa Zaatari ${ }^{1}$

${ }^{1}$ DZINE Partners

Published on: Jan 26, 2022

License: Creative Commons Attribution 4.0 International License (CC-BY 4.0). 


\section{$\underline{\text { RR:C19 Evidence Scale rating by reviewer: }}$}

- Reliable. The main study claims are generally justified by its methods and data. The results and conclusions are likely to be similar to the hypothetical ideal study. There are some minor caveats or limitations, but they would/do not change the major claims of the study. The study provides sufficient strength of evidence on its own that its main claims should be considered actionable, with some room for future revision.

$* * * * * * * * * * * * * * * * * * * * * * * * * * * * * * * * * * * * * * *$

\section{Review:}

The findings of this study confirm our current understanding of the most cost-efficient strategy to reduce SARS-COV-2 exposure risk, it aligns with ASHRAE core recommendations to 1 ) consider risk, energy, cost and comfort when deciding on a strategy or combination of mitigation strategies and 2) to have at least minimum outdoor air per applicable codes and Standards and MERV 13 and when applicable other control strategies.

This paper presents nicely results of energy modelling for a variety of climates for a large office building to compare equivalent outdoor air rate, energy cost per day, and normalised cost per air change rate presented for each month of the year. The results are clear and support the conclusions made by the authors. The manuscript adequately cites relevant literature but does not discuss sufficiently the limitations of the space type and few other parameters chosen for this modelling effort. Claims are generally supported by the data and the energy modelling used. Decision-makers should consider the claims in this study actionable with limitations based on the methods and data.

In general, there are grammatical and stylistic mistakes that need to be corrected before publishing. Figure 1 description and annotation needs some work for it to be useful. The term "ventilation" should be next to outdoor air and the term "recirculated air" should be next to the return air damper. The "purple" annotations that are supposed to indicate energy consumption are missing from the diagram. 